\title{
Pseudomembranous necrotizing laryngotracheobronchitis due to Mycoplasma pneumoniae: a case report and literature review
}

\author{
Wu Lei ${ }^{\dagger}$, Zhang Fei-Zhou ${ }^{\dagger}$, Chen Jing, Li Shu-Xian, Wu Xi-Ling and Tang Lan-Fang ${ }^{*}$
}

\begin{abstract}
Background: Pseudomembranous necrotizing laryngotracheobronchitis refers to an acute diffuse necrotizing inflammation in the mucosa of the larynx, trachea, and bronchus. It often occurs in infants and children having viral infections secondary to bacterial infections. Mycoplasma pneumoniae (M. pneumoniae) is a common pathogen that causes pneumonia in children. In recent years, serious complications due to M. pneumoniae infection, including necrotizing pneumonia, pulmonary embolism, and pleural effusion, have been increasingly reported.
\end{abstract}

Case presentation: An 11-year-old girl was admitted to our unit with cough, fever, and hoarseness persistent for a week. The results of the M. pneumoniae serological test, PCR examination with bronchial aspirate and bronchoalveolar lavage fluid (BALF), next-generation sequencing (mNGS) for BALF, all suggested the presence of M. pneumoniae infection. High-resolution CT scanning of the chest showed inflammation of the middle and lower lobes of the right lung. By bronchoscopy, the necrosis of the vocal cords, trachea, and bronchial mucosa was observed; each bronchial lumen contained a large amount of white viscous sputum. Pathological findings for bronchial mucosa suggested inflammatory necrosis. After administration of azithromycin and glucocorticoids, the symptoms of the patients were ameliorated. After 2 weeks post-discharge, the X-ray scan of her chest indicated the pneumonia resolution in the right lung.

Conclusions: In patients with pneumonia due to M. pneumoniae infection, which causes obvious hoarseness, bronchoscopy is necessary even if the lung lesions are not massively consolidated. When necrotizing lesions of the larynx, trachea, and bronchi are detected by bronchoscopy, the necrotic tissues in the corresponding parts should be conducted tissue biopsy for pathological examination. Apart from macrolide antibiotics, the administration of small doses of glucocorticoids is necessary.

Keywords: Pseudomembranous necrotizing laryngotracheobronchitis, Mycoplasma pneumoniae, Bronchoscopy, Glucocorticoid, Children

\section{Background}

Acute laryngotracheobronchitis can be classified into acute obstructive laryngotracheobronchitis and acute fibrinous laryngotracheobronchitis. The latter, which is

\footnotetext{
*Correspondence: 6195007@zju.edu.cn

tWu Lei and Zhang Fei-Zhou contributed equally to this work

Department of Pulmonology, The Children's Hospital, Zhejiang University

School of Medicine, National Clinical Research Center for Child Health,

3333 Binsheng Rd, Hangzhou 310052, Zhejiang, China
}

rarer than the former, is also called acute pseudomembranous necrotizing laryngotracheobronchitis and is a life-threatening illness [1]. The causes of pseudomembranous necrotizing laryngotracheobronchitis are mostly gram-positive bacterial infections (e.g., Staphylococcus aureus, Streptococcus hemolyticus, and Streptococcus pneumoniae) [2-4] that are secondary to viral infections (e.g., influenza virus, adenovirus), causing necrotizing damages to the larynx, trachea, and bronchial mucosa. In severe cases, falling off of the pseudomembrane, 
necrotizing mucus, and phlegm plugs, occur, that block the airway, thereby causing ventilatory dysfunction, eventually leading to breath failure and even death $[5,6]$.

Mycoplasma pneumoniae (M. pneumoniae) is a common pathogen responsible for respiratory tract infections in children. These present with various clinical manifestations, ranging from mild upper respiratory tract infections to severe necrotizing pneumonia [7]. Recently, with continuing in-detail studies on M. pneumoniae, researchers report that it not only causes severe lung diseases, such as necrotizing pneumonia, pulmonary embolism [8], pulmonary atelectasis [9], plastic bronchitis [10], and bronchiolitis obliterans [11] but also leads to extrapulmonary diseases, including meningoencephalitis [12], hepatitis [13], and pericarditis [14].

Reports on acute pseudomembranous necrotizing laryngotracheobronchitis secondary to $M$. pneumoniae infection are rather rare. To our knowledge, only two cases have been previously reported worldwide $[15,16]$. Herein, we report the case of treating a patient with $M$. pneumoniae-related acute pseudomembranous necrotizing tracheobronchitis. Our clinical experience may improve clinicians' understanding of the disease.

\section{Case presentation}

A previously healthy, 11-year-old girl was admitted to the hospital on September 17, 2021, with main complaints of persistent cough, fever, and hoarseness for a week. She was born at full-term, with vaginal delivery, and weighed
$3.75 \mathrm{~kg}$ at birth. Her parents had a non-consanguineous marriage. Neither any other associated diseases nor the use of any medicines was gathered.

The child had a persistent cough for a week. It was an irritating dry cough accompanied by a sore throat and hoarseness. There was no nasal congestion, runny nose, shortness of breath, canine barking cough, or dyspnea. She had a fever peaking at 40 degrees Celsius. The temperature decreased gradually to a normal level after oral administration of Ibuprofen, however, it would rise again after 3-4 h. There were no rashes or convulsions. She was referred to the outpatient clinic of our hospital. No obvious abnormalities were observed in the blood routine tests. The antigen tests for respiratory pathogens were also negative. She was diagnosed with "acute upper respiratory tract infection" and prescribed azithromycin tablets, $0.25 \mathrm{~g} /$ time, once/day orally for 2 days (Sept. 10 Sept. 11). However, her coughing worsened, manifesting through a marked increase in frequency, and she could not sleep well at night. Routine blood tests were performed in the second outpatient clinic which showed that both white blood cell counts $\left(14.13 \times 10^{9} / \mathrm{mL}\right)$ and the high-sensitivity C-reactive protein $(23.56 \mathrm{mg} / \mathrm{L})$ levels were elevated. In addition, the chest radiograph showed a fuzzy patchy shadow on the right lower lung on Sept. 12 (Fig. 1A). This was considered a case of "acute bronchopneumonia" and for clearing the infection, Cefotaxime Sodium $3 \mathrm{~g} /$ time, once/day for 2 days (Sept. 12-Sept. 13), followed by Ceftriaxone sodium at $2 \mathrm{~g} /$ time, once/day

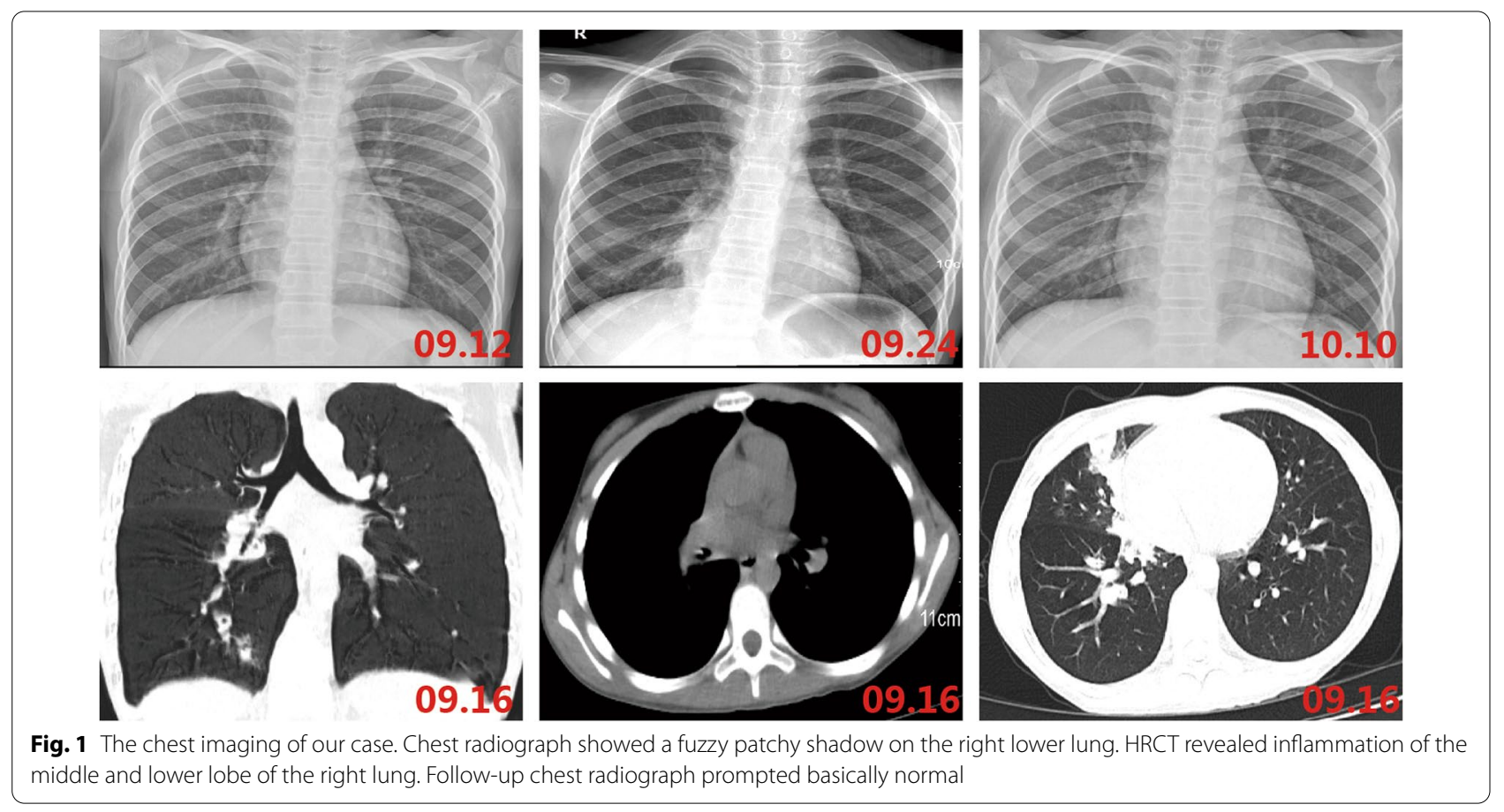


for 3 days (Sept. 14-Sept. 16), were administered. Her cough and fever did not show any significant improvement and she returned to our hospital for follow-up visits. A repeated, routine blood test showed that the white blood cell counts were normal $\left(6.82 \times 10^{9} / \mathrm{mL}\right)$, but the high-sensitivity C-reactive protein $(34.15 \mathrm{mg} / \mathrm{L})$ levels were still high. A High-resolution CT scan of the chest (HRCT) showed inflammation of the middle and lower lobes of the right lung (Fig. 1D-F). She was diagnosed with "pneumonia" and azithromycin $0.28 \mathrm{~g} /$ time, once/ day was intravenously administered for a day (Sept.16). Her cough improved a little, but the fever was recurrent. For further diagnosis and treatment, she was admitted to our unit with "pneumonia" on Sept. 17.

Physical examinations suggested that the tonsils had a second-degree enlargement and were covered with white secretion. Wet rales were heard from the right lung and there were no obvious abnormalities in the heart, abdomen, skin, and/or the nervous system.

Increased levels of high-sensitivity $\mathrm{C}$-reactive protein (49.39 $\mathrm{mg} / \mathrm{L})$, alanine aminotransferase $(56 \mathrm{U} / \mathrm{L})$, and lactate dehydrogenase $(388 \mathrm{U} / \mathrm{L})$ were observed. The results of serological tests for M. pneumoniae, PCR examination for bronchial aspirate and BALF, and mNGS of BALF, all suggested the presence of $M$. pneumoniae infection. All results of laboratory tests are listed in Table 1 . There were no obvious abnormalities in the electrocardiogram for the heart and the abdominal color Doppler ultrasound.

After admission, azithromycin (Sept.17-Sept. 22) for anti-infection, methylprednisolone (Sept.20-Sept.22) for anti-inflammation, and Compound Glycyrrhizin (Sept.18-Sept.20) for liver protection effects were administered. Bronchoscopy was performed on Sept. 20 and obviously swollen left vocal cords and white stripes at the lower edge of the right vocal cords were observed. Moreover, ulcers were observed in the upper and middle trachea, with local depressions, abundant yellow-white secretions on the surface, and smaller granulation-like tissues. Moreover, in each bronchial lumen, abundant yellow and white viscous secretions were observed (Fig. 2). The pseudomembranes were removed and bronchoalveolar lavage (BAL) was performed. Pathology through the tracheal mucosal biopsy showed cellulose exuding, chronic mucosal inflammation, and inflammatory necrotic tissues (Fig. 3). Finally, the body temperature returned to normal levels on Sept 20, coughing decreased on the fourth day, and hoarseness improved by Sept 22. By Sept 24, her body temperature was normal for continuous $48 \mathrm{~h}$; occasionally coughing but no hoarseness was noted. The chest radiograph demonstrated the presence of right lower lung pneumonia (Fig. 1B). She was discharged (Fig. 4). On Oct. 10, her chest radiograph was found to be back to normal (Fig. 1C).

\section{Discussion and conclusions}

Pneumonia caused by M. pneumoniae occurs mainly in adolescents, the elderly, immunocompetent individuals, and older children. The clinical manifestations include an irritating cough [17]. In recent years, the proportion of refractory M. pneumoniae pneumonia cases has been on a gradual rise. An 11-year-old girl presented fever and cough and was diagnosed with pneumonia upon imaging. She was positive for IgM and RNA of M. pneumoniae. Thus, the diagnosis of $M$. pneumoniae pneumonia was confirmed.

Table 1 The sequence of her laboratory test results as a hospital inpatient

\begin{tabular}{|c|c|c|c|c|c|c|c|}
\hline & 17 Sept & 18 Sept & 19 Sept & 20 Sept & 21 Sept & 22 Sept & 23 Sept \\
\hline White blood cell $\left(4-12 \times 10^{9} / \mathrm{mL}\right)$ & 8.36 & & & & 9.94 & & \\
\hline High-sensitivity C-reactive protein ( $0-8 \mathrm{mg} / \mathrm{L})$ & 49.39 & & & & 12.67 & & \\
\hline D-dimer $(<0.55 \mathrm{mg} / \mathrm{L})$ & & 3.97 & & & 3.96 & & \\
\hline Alanine aminotransferase (<50 U/L) & & 56 & & & 38 & & \\
\hline M. pneumoniae RNA of bronchial aspirate & & & Positive & & & & \\
\hline M. pneumoniae RNA of BALF & & & & & & & Positive \\
\hline Epstein-Barr virus antibody and DNA & & & & Negative & & & \\
\hline $\mathrm{MP}+\mathrm{CP}+\mathrm{LG}$ antibody & & & & Negative & & Negative & \\
\hline Tuberculosis infection T cell detection & & & & $\lg$ 5.32 & & & \\
\hline Lactate dehydrogenase (110-295 U/L) & & 388 & & & 258 & & \\
\hline Procalcitonin $(0-0.46 \mathrm{ng} / \mathrm{mL})$ & 0.273 & & & & & & \\
\hline Erythrocyte sedimentation rate $(0-20 \mathrm{~mm} / \mathrm{h})$ & 50 & & & & & & \\
\hline Blood culture & & & & & Negative & & \\
\hline BALF culture & & & & & Negative & & \\
\hline Tuberculosis smear examination of BALF & & & & & & & Negative \\
\hline
\end{tabular}




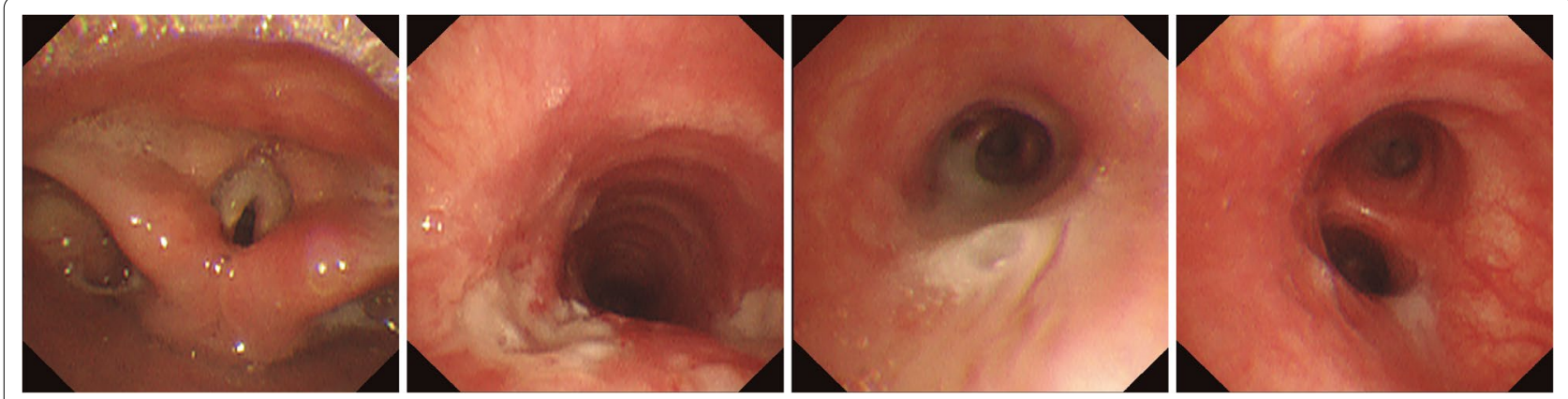

Fig. 2 The larynx, trachea, and bronchi under bronchoscopy. Obviously swollen left vocal cords, white strips at the lower edge of the right vocal cords were observed. Moreover, ulcers were observed in the upper and middle trachea, with local depressions, more yellow-white secretions on the surface, and smaller granulation-like tissues
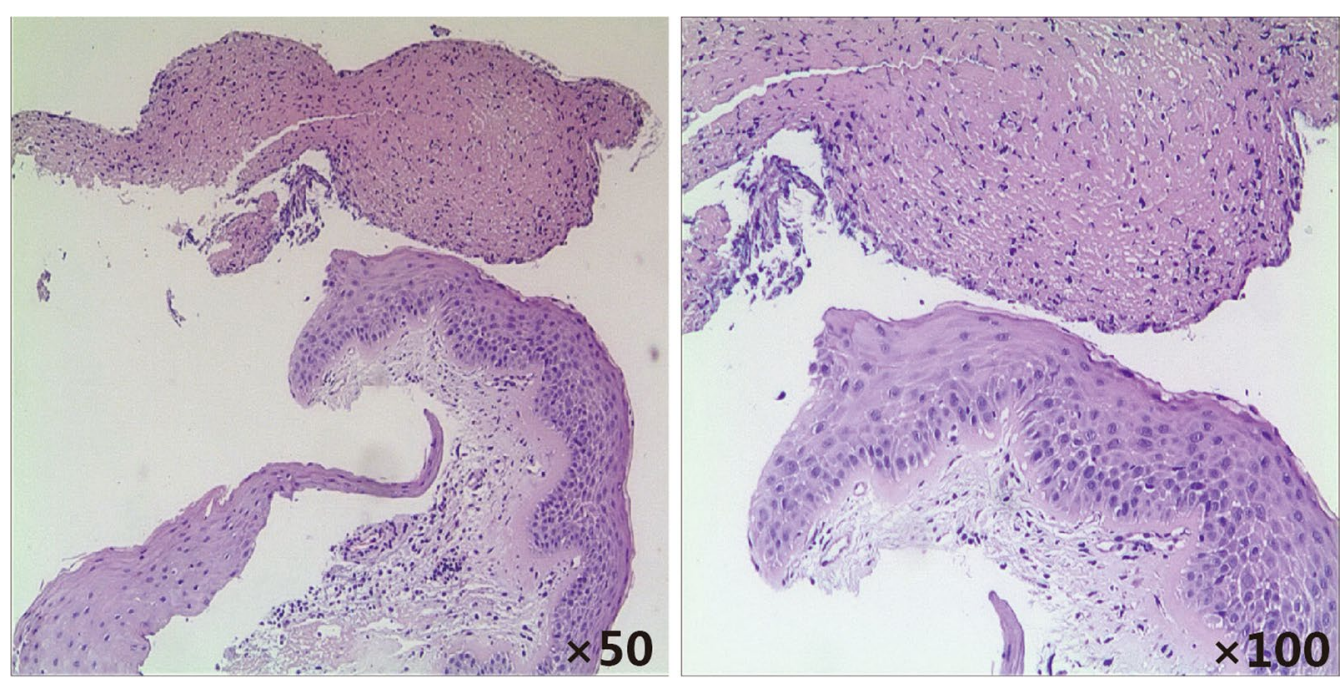

Fig. 3 Hematoxylin-eosin staining of clamped tracheal mucosa. Pathology reveals exuding cellulose, chronic mucosal inflammation, and inflammatory necrotic tissues

Obvious hoarseness in this patient caught our attention and we decided to perform bronchoscopy and mNGS of the BALF for further confirmation. By bronchoscopy, obvious pseudomembranes were observed in the trachea. The pathological results were in line with our expectations, that is, the presence of necrotizing bronchitis. The mNGS of BALF suggested the presence of $M$. pneumoniae infection only and no other pathogens were detected. The diagnosis of $M$. pneumoniae-related acute pseudomembranous necrotizing laryngotracheobronchitis was confirmed. To our knowledge, only two other cases of $M$. pneumoniae-causing pseudomembranous necrotizing laryngotracheobronchitis have been reported previously (Table 2). Both these patients presented with cough and fever. Notably, case 2 was also that of a child, and in addition to cough and fever, obvious hoarseness was also present. Hence, in patients with M. pneumoniae pneumonia, having obvious hoarseness, acute pseudomembranous necrotizing laryngotracheobronchitis should be considered during the differential diagnosis.

As acute pseudomembranous necrotizing laryngotracheobronchitis is relatively rare, the underlying mechanisms remain unclear. Accumulating data show that the initiating factors of acute pseudomembranous necrotizing laryngotracheobronchitis are viral infections. Subsequent bacterial infections are mostly caused by the Gram-positive bacteria (e.g., Staphylococcus aureus) [2-4]. Although leukotoxin crucially contributes to Staphylococcus aureus-induced necrotizing laryngotracheobronchitis and pneumonia, owing to the destruction of the airway epithelial cells and lungs [18], the mechanisms underlying $M$. pneumoniae-induced necrotizing 


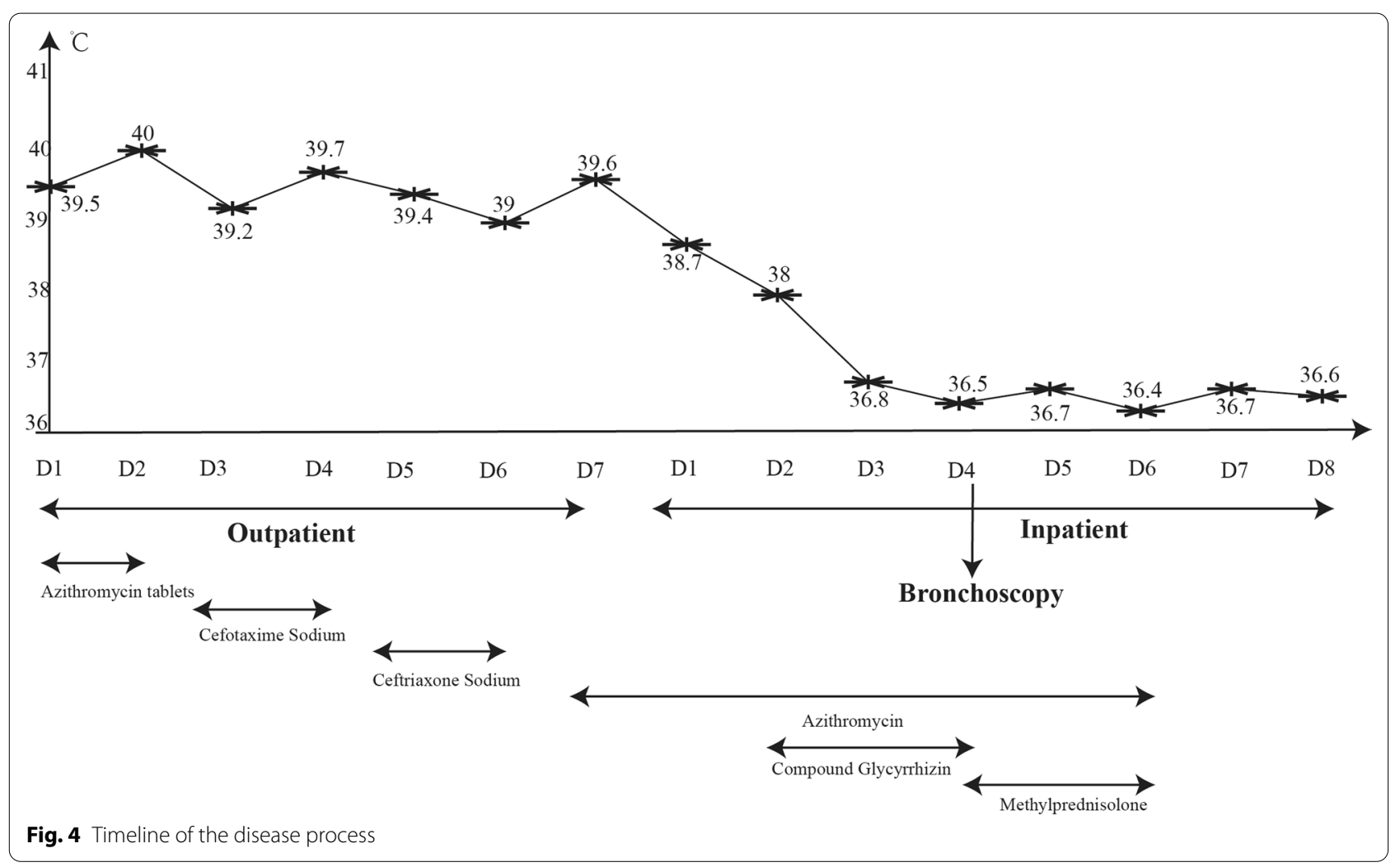

Table 2 Acute pseudomembranous necrotizing laryngotracheobronchitis associated with M. pneumoniae infection

\begin{tabular}{|c|c|c|c|c|c|c|c|c|}
\hline Cases & Sex & Age (years) & Chief complaints & $\begin{array}{l}\text { M.pneumoniae } \\
\text { detection }\end{array}$ & Treatment & $\begin{array}{l}\text { Bronchoscopy } \\
\text { times }\end{array}$ & $\begin{array}{l}\text { Admission } \\
\text { days }\end{array}$ & Outcome \\
\hline $1^{15}$ & $\mathrm{~F}$ & 29 & $\begin{array}{l}\text { Cough, fever, sore } \\
\text { throat }\end{array}$ & $P C R+\lg M$ & $\begin{array}{l}\text { Moxifloxacin, corticos- } \\
\text { teroid }\end{array}$ & 1 & 7 & Recover \\
\hline $2^{16}$ & $\mathrm{~F}$ & 2 & $\begin{array}{l}\text { Cough, hoarseness, } \\
\text { dyspnea }\end{array}$ & $m N G S+\lg M$ & $\begin{array}{l}\text { Azithromycin, ceftri- } \\
\text { axone, methylpredni- } \\
\text { solone }\end{array}$ & 3 & 21 & Recover \\
\hline Current case & $\mathrm{F}$ & 11 & $\begin{array}{l}\text { Cough, fever, hoarse- } \\
\text { ness }\end{array}$ & $P C R+\lg M+m N G S$ & $\begin{array}{l}\text { Azithromycin, methyl- } \\
\text { prednisolone }\end{array}$ & 1 & 8 & Recover \\
\hline
\end{tabular}

$P C R$ polymerase chain reaction, $F$ female

laryngotracheobronchitis and pneumonia have not yet been reported. These may possibly be related to the direct destruction of the trachea and bronchial mucosa by M. pneumonia, the toxic changes in tracheal epithelial cells, and excessive immune responses of the body, similar to the mechanism of action of M. pneumoniae-associated necrotizing pneumonia [19].

Although three patients with M. pneumoniae infection-related acute pseudomembranous necrotizing laryngotracheobronchitis have been reported to recover, necrotizing tissue, phlegm plugs, and pseudomembrane may fall off and block the airway, thereby causing a critical situation. In patients with M. pneumoniae infections, recognizing the complications outside the lung is equally essential in addition to the lung injury. For acute pseudomembranous necrotizing laryngotracheobronchitis, bronchoscopy is a crucial method to diagnose and remove pseudomembranes, thereby alleviating airway obstruction $[4,20]$. All three cases of M. pneumoniae infection-related acute pseudomembranous necrotizing laryngotracheobronchitis patients have reportedly undergone bronchoscopy, which further confirmed the diagnosis and were treated by the removal of the pseudomembranes and for BAL. In children with M. pneumoniae infection and unexplained symptoms (e.g., unrelieved hoarseness, persistent fever), bronchoscopy 
should be performed for further investigation. Removing the pseudomembranes and washing out the bronchial lumen may improve their condition.

Recently, it has been proposed that the severe inflammatory reactions after $M$. pneumoniae infection are caused mainly by abnormal immune responses. Therefore, in patients who rapidly progress in the acute stage, severe cases, and those with extrapulmonary complications, early application of glucocorticoids should be considered apart from macrolides administration, to quickly relieve symptoms, shorten the course of the disease, and increase the cure rate [21]. As in the two previously reported cases, methylprednisolone $(1 \mathrm{mg} / \mathrm{kg} /$ day $)$ was administered to our patient on the second day of admission. The fever, cough, and hoarseness of the child were significantly relieved.

In conclusion, in $M$. pneumoniae pneumonia patient with obvious hoarseness, acute pseudomembranous necrotizing laryngotracheobronchitis should be considered in the differential diagnosis; bronchoscopy and BAL should be performed early during the course of treatment. Besides macrolide antibiotics, glucocorticoids should be administered in these refractory cases.

\section{Abbreviations \\ M. pneumonia: Mycoplasma pneumonia; HRCT: High-resolution CT; BAL: Bronchoalveolar lavage; BALF: Bronchoalveolar lavage fluid; mNGS: Next- generation sequencing.}

\section{Acknowledgements}

We thank the patient and her parents for permitting us to use the data. We would like to thank Bullet Edits Limited for the linguistic editing and proofreading of the manuscript.

\section{Authors' contributions}

WL and ZFZ wrote the first draft of the manuscript; CJ, LSX, and WXL participated in the data collection and improved the subsequent draft of the article. TLF revised the manuscript to ensure authenticity and practicability. All authors have read and approved the final manuscript as submitted and agree to accountability for all aspects of this work. All authors read and approved the final manuscript.

\section{Funding}

This work was supported by Grants from the National Natural Science Foundation of China (81170016, 81470214 and 82070028) and the Zhejiang Provincial Program for the Cultivation of High-Level Innovative Health Talents (2016). The funding supported the cost of metagenomic next-generation sequencing. The funding was not used in any other aspect of the study, including its design, collection of data, writing of the report, or decision to submit for publication.

\section{Availability of data and materials}

All data generated or analyzed during this study are included in this published article [and the additional information files].

\section{Declarations}

\section{Ethics approval and consent to participate}

This study design was approved by the ethics committee of the Children's Hospital affiliated to the Zhejiang University School of Medicine and a signed informed consent form was obtained from the patient's parents.

\section{Consent for publication}

The patient's parents have provided informed consent for publication. And the parents gave their written consent for their child's personal or clinical details along with any identifying images to be published in this study.

\section{Competing interests}

The authors declare no competing interests.

Received: 25 November 2021 Accepted: 15 February 2022

Published online: 23 February 2022

\section{References}

1. Szpunar J, Glowacki J, Laskowski A, Miszke A. Fibrinous laryngotracheobronchitis in children. Arch Otolaryngol. 1971;93:173-8.

2. Gaugler C, Astruc D, Donato L, Rivera S, Langlet C, Messer J. Neonatal necrotizing tracheobronchitis: three case reports. J Perinatol. 2004;24:259-60.

3. Takahashi S, Nakamura M. Necrotizing tracheobronchitis caused by influenza and Staphylococcus aureus co-infection. Infection. 2018:46:737-9.

4. Wu X, Wu L, Chen Z. The important role of endoscopy in management of pediatric pseudomembranous necrotizing tracheitis. Front Pediatr. 2020:8:360.

5. Tsokos M, Zöllner B, Feucht HH. Fatal influenza A infection with Staphylococcus aureus superinfection in a 49-year-old woman presenting as sudden death. Int J Legal Med. 2005;119:40-3.

6. Park SS, Kim SH, Kim M, et al. A case of severe pseudomembranous tracheobronchitis complicated by co-infection of influenza A (H1N1) and Staphylococcus aureus in an immunocompetent patient. Tuberc Respir Dis. 2015;78:366-70.

7. Zheng B, Zhao J, Cao L. The clinical characteristics and risk factors for necrotizing pneumonia caused by Mycoplasma pneumoniae in children. BMC Infect Dis. 2020;20:391.

8. Chen S, Ding Y, Vinturache A, Gu H, Lu M, Ding G. Pulmonary embolism associated with mycoplasma in a child. Lancet Infect Dis. 2020;20:1347.

9. Su DQ, Li JF, Zhuo ZQ. Clinical analysis of 122 cases with mycoplasma pneumonia complicated with atelectasis: a retrospective study. Adv Ther. 2020;37:265-71

10. Zhong H, Yin R, Zhao R, Jiang K, Sun C, Dong X. Analysis of clinical characteristics and risk factors of plastic bronchitis in children with Mycoplasma pneumoniae pneumonia. Front Pediatr. 2021;9:735093.

11. Xu W, Yang H, Liu H, et al. Bronchoalveolar lavage T cell cytokine profiles and their association with lung function in children with Mycoplasma pneumoniae-associated bronchiolitis obliterans. Pediatr Pulmonol. 2020;55:2033-40.

12. Özel C, Dafotakis M, Nikoubashman O, Litmathe J, Matz O. Schöne U [Mycoplasma pneumoniae-induced meningoencephalitis]. Fortschr Neurol Psychiatr. 2015;83:392-6.

13. Poddighe D. Mycoplasma pneumoniae-related hepatitis in children. Microb Pathog. 2020;139:103863.

14. Vijay A, Stendahl JC, Rosenfeld LE. Mycoplasma pneumoniae pericarditis. Am J Cardiol. 2019;123:1383-4.

15. Lee $\mathrm{YH}$, Seo H, Cha SI, Kim CH, Lee J. A case of pseudomembranous tracheitis caused by Mycoplasma pneumoniae in an immunocompetent patient. Ann Transl Med. 2019;7:205.

16. Wen SH, Lin L, Yu G, Xu CF, Zhang HL, Zheng YM. Pseudomembranous laryngotracheobronchitis due to coinfection with human bocavirus 1 and Mycoplasma pneumoniae: a case report. Transl Pediatr. 2021;10:673-8

17. Lanao AE, Chakraborty RK, Pearson-Shaver AL. Mycoplasma Infections. StatPearls. Treasure Island (FL): StatPearls Publishing Copyright (c) 2021, StatPearls Publishing LLC.; 2021.

18. Morgan M. Staphylococcus aureus, Panton-Valentine leukocidin, and necrotising pneumonia. BMJ (Clinical research ed). 2005;331:793-4.

19. He J, Liu M, Ye Z, et al. Insights into the pathogenesis of Mycoplasma pneumoniae (Review). Mol Med Rep. 2016;14:4030-6. 
20. Lu S, Liu J, Cai Z, Shuai J, Huang K, Cao L. Bronchial casts associated with Mycoplasma pneumoniae pneumonia in children. J Int Med Res. 2020;48:300060520911263.

21. Kim HS, Sol IS, Li D, et al. Efficacy of glucocorticoids for the treatment of macrolide refractory mycoplasma pneumonia in children: meta-analysis of randomized controlled trials. BMC Pulm Med. 2019;19:251.

\section{Publisher's Note}

Springer Nature remains neutral with regard to jurisdictional claims in published maps and institutional affiliations.

- fast, convenient online submission

- thorough peer review by experienced researchers in your field

- rapid publication on acceptance

- support for research data, including large and complex data types

- gold Open Access which fosters wider collaboration and increased citations

- maximum visibility for your research: over $100 \mathrm{M}$ website views per year

At $\mathrm{BMC}$, research is always in progress.

Learn more biomedcentral.com/submissions 